Artículo

\title{
Agricultura intensiva y calidad de suelos: retos para el desarrollo sustentable en Sinaloa
}

\author{
Aldo Alan Cuadras-Berrelleza ${ }^{1}$ \\ Víctor Manuel Peinado-Guevara ${ }^{1 \S}$ \\ Héctor José Peinado-Guevara ${ }^{1}$ \\ José de Jesús López-López ${ }^{1}$ \\ Jaime Herrera-Barrientos ${ }^{2}$ \\ ${ }^{1}$ Facultad de Ciencias Económicas y Administrativas-Universidad Autónoma de Sinaloa. Blvr. Juan de Dios \\ Batiz, Col. San Joachin, Guasave, Sinaloa. CP. 81101. (aalan@uas.edu.mx; hpeinado75@hotmail.com; \\ jesuslope4@gmail.com). ${ }^{2}$ Centro de Investigación Científica y de Educación Superior de Ensenada. \\ Carretera Ensenada-Tijuana núm. 3918, zona Playitas, Ensenada, Baja California. CP. 22860. \\ (jherrera@ cicese.mx). \\ $\S^{\S}$ Autor para correspondencia: v_peinado@ hotmail.com.
}

\section{Resumen}

En una dimensión regional, se analizan dos factores claves de la actividad agrícola de Guasave, Sinaloa, México que influyen directamente en el desarrollo rural sustentable: la agricultura intensiva y la calidad de suelos. La presente investigación se diseñó como un estudio cualitativo de tipo descriptivo de este ramo productivo, para ello se aplicaron 164 entrevistas a productores y se analizaron diversos documentos con información del sector, tales como informes técnicos y boletines. Se encontró que la agricultura intensiva predomina en el área de estudio, con prácticas y patrones de producción de tipo tradicional, como son sistemas de riego de gravedad y uso desmedido de agroquímicos, lo cual presupone manejos agrarios que van en contra del contexto sustentable. Se requiere de investigación-acción para la implementación de estrategias de resiliencia con un enfoque sostenible, alineadas a la gobernanza ambiental adecuada de la actividad y a las necesidades del sector, toda vez que se siguen observando problemas como la pérdida de fertilidad de los suelos, un incremento en la salinidad y erosión en algunos predios agrícolas.

Palabras clave: desarrollo rural sustentable, patrones de producción, práctica agrícola.

Recibido: octubre de 2021

Aceptado: diciembre de 2021 


\section{Introducción}

La agricultura ha sido históricamente reconocida por sus aportes a la alimentación y al desarrollo de los pueblos, hoy en día, su contribución a la economía, aprovisionamiento de alimentos y relación con el equilibrio sustentable invita al debate y discusión sobre los efectos que los sistemas de producción pueden generar hacia la sociedad, medio ambiente y entorno económico. El aporte a la seguridad alimentaria y el impacto al desarrollo sostenible que esa actividad conlleva es, y seguirá siendo, un factor determinante para mantener un equilibrio ambiental y social (Viana et al., 2022). Laurett et al. (2021) sostienen que tanto la falta de información y conocimiento, así como la de planificación y apoyo económico dificultan los propósitos de implementar una agricultura sustentable.

La transición hacia modelos agrícolas sostenibles en la agricultura y los sistemas alimentarios es un reto complejo que demanda de acciones políticas, jurídicas y culturales con la participación coactiva de las partes interesadas, comunidad científica y de innovación tecnológica López et al. (2021). Por otro lado, pero apegado a lo anterior, las comunidades de campesinos forman uno de los pilares básicos que sostienen la actividad económica de diversas regiones del mundo, no solo por suministrar alimentos, sino por ser un grupo socioeconómico significativo que provee bienes de primera necesidad a las zonas rurales y urbana.

Por ello, la importancia de los grupos de población rural y su bienestar dependerá en gran medida de lo bien o mal que puedan llevarse a cabo las actividades del campo. Cómo ejemplo de lo antes planteado, la década de los años 80's se caracterizó por el actuar de las comunidades campesinas, las cuales se tuvieron que ajustar a la situación económica, definiéndose nuevas maneras de organizarse socialmente, de acuerdo con el panorama nuevo del país y de su política económica (González-Jácome, 2004). De tal forma, que, los grupos de comunidades rurales tendrán hoy en día que adaptarse al entorno que el sector mismo ha propiciado, implementando medidas y programas de acción en conjunto con gobierno y demás actores del sector (políticas, aspectos económicos, comerciales, entre otros), siendo resilientes y holísticos a la vez.

La agricultura en México es un sector con representaciones menores en términos de producto interno bruto (PIB), y en términos económicos totales a la baja, por ejemplo, en 2009 representó cerca de $4 \%$ del PIB. Sin embargo, la agricultura provee empleo aproximadamente a $13 \%$ de la fuerza de trabajo, lo que representa alrededor de 3.3 millones de agricultores, 4.6 millones de trabajadores asalariados y familiares no remunerados. Tiene más relación con el desarrollo territorial, aproximadamente $24 \%$ de la población total vive en zonas rurales (Fox y Haight, 2010).

Existen varios estudios sobre la problemática ambiental, que imperan en la agricultura del estado de Sinaloa, destacando dentro de estos, aquellos relacionados con el uso del agua en la agricultura, uso desmedido de agroquímicos en el sector, impacto ambiental como producto de las prácticas agrícolas, contaminación en el sector, entre otros (Norzagaray-Campos et al., 2010; PeinadoGuevara et al., 2015; Valenzuela-Martínez et al., 2019; Cruz-Delgado y Leos-Rodríguez, 2019).

En cuanto al suelo y su capacidad de producir y servicios que presta al ecosistema, se evalúa midiendo un grupo mínimo de datos que corresponden a diversas propiedades físicas, químicas y biológicas, Baveye et al. (2016); (Vallejo-Quintero, 2013). Este recurso natural ha sido muy 
importante para la población de diversas regiones del mundo, principalmente por su relevancia asociada a la producción de alimentos; sin embargo, a lo largo de la historia se ha venido presentando distintos problemas, según el Informe de la Situación del Medio Ambiente en México publicado por la Comisión Nacional Forestal y la Universidad Autónoma de Chihuahua (CONAFOR y UACH, 2013).

Los problemas de degradación del suelo son de tipo físico, químico, eólico, hídrico, entre otros, donde la agricultura ha sido parte de estos. Al respecto, estudios como el de Valenzuela-Martínez et al. (2019) muestran que la actividad agrícola del estado de Sinaloa genera diversos problemas ambientales, entre los que destaca la contaminación de los suelos, atribuida principalmente al uso de agroquímicos. Además, a pesar de ser el sostén de muchas de las economías del mundo, la agricultura se encuentra bajo una creciente presión de deterioro derivada tanto del crecimiento poblacional como de los patrones insostenibles de producción y consumo globales. Díaz et al. (2017) señalan que la agricultura ha sido determinante en los problemas que se tienen actualmente en los suelos y se relacionan con el uso de insumos químicos de manera intensiva.

De acuerdo con (CONAFOR y UACH, 2013) en un informe final sobre la degradación de tierras y desertificación, aproximadamente 35\% del territorio nacional muestra problemas de erosión eólica. Sinaloa y Sonora son de los estados donde se presentan diversas complicaciones relacionadas con el uso del suelo, como lo son: salinidad, alcalinidad, sodicidad, degradación física, entre otros problemas.

La agricultura sinaloense se ha consolidado como la más importante del país, por su alta capacidad para producir alimentos, pero también como generadora de empleos y divisas. Guasave, considerado como el corazón agrícola de México, es uno de los distritos altamente productores, está localizado al Noroeste del Estado (Gobierno de Sinaloa, 2019), y sustenta su desarrollo principalmente en las actividades primarias, sobre todo, en la agrícola, actividad que se respalda con el uso de alta tecnología en los procesos productivos (Gobierno municipal de Guasave, 2019) destacando en la producción de granos y hortalizas (CODESIN, 2019; SIAP, 2020).

Por lo antes indicado, se plantea como objetivo 'analizar la influencia que ejerce la agricultura intensiva sobre la sustentabilidad y la calidad de suelos en el valle agrícola del municipio de Guasave, y que impacta directamente en el desarrollo rural sustentable'. Lo que motiva estar en la búsqueda constante de las mejoras del sector, generando conocimiento vanguardista e innovador que permita solucionar las demandas de este, de la sociedad y del medio ambiente, a través de procesos ecosistémicos, integrales y sustentables.

\section{Agricultura intensiva y prácticas agrícolas}

La actividad agrícola tiene un impacto directo en la calidad y formas de vivir de los campesinos, los sistemas de producción están influenciadas por las políticas de modernización del sector agropecuario, los proyectos de desarrollo de los gobiernos, la industrialización, el crecimiento desmedido de las áreas urbanas y otros procesos propios de las sociedades en progreso (González et al., 2007). Los conocimientos y prácticas agrícolas que se han originado y adaptado a través de la historia han sido causa del desarrollo; conocimientos que ha pasado de generación a generación, de padres a hijos, formando un cúmulo de riquezas que permiten adecuar y organizar, de la mejor manera posible, el trabajo del campo agrícola. 
Dentro de estas se encuentran el conocimiento sobre el medio ambiente y agrícola, sobre el uso del agua, suelo, lluvia, entre otros (Pérez-Sánchez et al., 2014). Investigadores como TamayoManrique et al. (2016) hacen hincapié en que los conocimientos de tipo tradicional de la agricultura ya son del pasado para los productores de hoy, apuntando que este tipo de actividad, ha quedado poco a poco en el olvido, debido a que fue sustituida por la aplicación y uso de las nuevas tecnologías. La incorporación tecnológica aunada a prácticas agrícolas convencionales y el uso de agroquímicos, han propiciado la desertificación de los suelos, contaminación del aire y del ambiente, entre otros (Chalán, 2019).

De acuerdo con Castillo-Valdez et al., (2021) 'las prácticas de manejo del suelo alteran sus propiedades, especialmente cuando las entradas de energía al sistema suelo-planta son menores que las salidas, o su resiliencia es incapaz de regresarlo al estado previo a la intervención'. La Organización de las Naciones Unidas para la Alimentación y la Agricultura (FAO), define al manual de buenas prácticas agrícolas como un grupo de actividades y acciones que darán pie al actuar bajo principios, normas y recomendaciones técnicas que se pueden ajustar a los sistemas de producción, procesamiento y transporte de alimentos con la finalidad de preservar, de la mejor manera posible, la salud humana, cuidar al medio ambiente y a la sociedad (FAO, 2012a).

Dada la variabilidad climática que se experimenta en nuestros días, las buenas prácticas agrícolas constituyen una herramienta indispensable para el manejo de riesgos. Su aplicación; sin embargo, implica fomentar la innovación, aumentar el conocimiento y proveer una visión holística a las partes interesadas, en particular a los pequeños productores, para que puedan mejorar sus sistemas productivos, incrementar su resiliencia y asegurar su sustentabilidad (Díaz et al., 2017).

En la actualidad, las prácticas agrícolas siguen dando de qué hablar y son factor de análisis para los investigadores que se identifican dentro de las líneas relacionadas con el cuidado del medio ambiente y el bienestar de la sociedad. Es muy común que los grupos de agricultores y campesinos pongan en práctica una serie de actividades agrícolas con las que buscan adaptarse a las propiedades de cada unidad de suelo, tales propiedades generalmente muestran cambios negativos como un deterioro de este importante recurso, situación que es originada por el uso inapropiado, prolongado y/o intensivo de agroquímicos (Zinck et al., 2005). Por su parte, Castillo et al. (2020) señalan que la actividad agrícola intensiva, aunada a prácticas agrícolas comunes, como los monocultivos y aplicación de agroquímicos, ha generado problemas en la calidad de los suelos.

La calidad de los suelos son los atributos o características que lo hacen ser de un modo u otro; es decir, apto o no para ciertas actividades. Aquí, por calidad de suelo se entiende las características, atributos o propiedades, por ejemplo, las fisicoquímicas y microbiológicas que permitan al suelo ser fértil y producir productos sanos (Andrade et al., 2021). Entonces, la calidad de los suelos es un factor determinante en la producción de alimentos sanos, por lo que su deterioro, afecta al medio ambiente, la economía y el bienestar social. Estos problemas han sido generados por la falta de conciencia social que se ha observado en las últimas décadas, aunado a la falta de leyes sólidas y normas que regulen y vigilen el adecuado uso de este recurso en las actividades donde más se explota, llevando al desgaste y empobrecimiento de los suelos en países como México (Cámara de Diputados y CEDRSSA, 2019). 


\section{Materiales y métodos}

Se parte de un análisis descriptivo bajo un enfoque cualitativo, cuyas variables de análisis fueron agricultura, calidad de suelos y sustentabilidad. Se tomó como referencia el entorno poco sustentable que se observa y predomina en las prácticas agrícolas cotidianas, así como en patrones de producción de los productores del valle agrícola del municipio de Guasave, Sinaloa, México. Para determinar el tamaño de la muestra, se consideró un universo de 12446 agricultores registrados en el programa producción para el bienestar (antes Programa de Apoyos Directos al Campo: Procampo) a la cual se le aplicó la ecuación estadística para proporciones poblacionales y considerando un margen de error de 10\% con un nivel de confianza de 99\%, arrojó un total de 164 productores, los cuales fueron seleccionados de forma no aleatoria (Reus González et al., 2021).

A la población objeto de estudio, se le aplicó una entrevista estructurada (Arias, 2012). Se evaluaron como variables: las principales actividades agrícolas, los patrones de producción, el uso de agroquímicos, el desarrollo rural, la calidad de suelos, entre otras determinantes, para lo cual, se diseñó una guía de preguntas alineadas al objetivo de la investigación; asimismo, se tomaron como referencia datos descriptivos del entorno agrícola e información directa del actor estudiado, así como de sus actividades observables (Taylor y Bogdan, 1984).

\section{Resultados y discusión}

En la entrevista aplicada a los agricultores seleccionados, se cuestionó sobre el quehacer en sus predios agrícolas y el tiempo que dedican a estas actividades, se encontró que $48.77 \%$ dedica de 1 a $4 \mathrm{~h}$ de actividad diaria a sus predios agrícolas, $27.16 \%$ de 4 a $7 \mathrm{~h}$ diarias, $10.49 \%$ de 8 a $10 \mathrm{~h}$, $12.35 \%$ dice dedicarle tiempo completo a esta actividad, mientras que $1.23 \%$ de la población no supo estimar que tiempo emplea a dicha actividad. Es preciso señalar que $61.74 \%$ de la población objeto de estudio siembra entre 1 y 10 ha, predominando la siembra de maíz, frijol y sorgo en granos y de tomate, chile y calabaza en hortalizas.

En cuanto a la práctica agrícola relacionada con el uso de los recursos hídricos para el riego parcelario, $90 \%$ de la población recurre al sistema de gravedad mediante canales de tierra, mientras que $10 \%$ emplean otros sistemas, como aquellos que se valen del uso de tecnologías de riego como son: aspersión, microaspersión y goteo. En este apartado, es importante señalar que 69.75\% del agua que se utiliza, proviene de las presas de la región, ésta se identifica como agua blanca o cruda, mientras que $7.41 \%$ utiliza aguas tratadas, el resto desconoce el tipo de agua que usa.

Lo anterior muestra que el riego por gravedad sigue siendo una práctica predominante para los agricultores, la fuerte dependencia del agua que se acumula de forma natural en las presas, indica el origen de un grave problema, sobre todo, en temporadas de sequías, lo que pone de manifiesto, la falta de infraestructura, programas de apoyo y de tecnologías eficientes en el uso de este recurso.

En el Cuadro 1, se muestran las prácticas agrícolas de manera general y que predominan en la región, se puede observar que el agricultor sigue presentando preferencias por actividades acordes a su conocimiento tradicional, encontrando aquellas que dañan directamente los recursos como el suelo, sin que signifique esto, que el daño causado sea por mala intención, sino más bien por falta de conocimiento al respecto, como en el uso de fertilizantes químicos, herbicidas e insecticidas 
químicos; mientras que, se tienen niveles de incidencia bajas en aquellas prácticas consideradas sustentables, tales como: uso de semillas criollas, abono natural, biofertilizantes, herbicidas e insecticidas orgánicos, estudios de suelo, certificaciones orgánicas, principalmente.

Cuadro 1. Prácticas agrícolas más comunes del agricultor del municipio de Guasave.

\begin{tabular}{ccc}
\hline Número & Práctica agrícola & $(\%)$ \\
\hline 1 & Fertilizantes químicos & 82.72 \\
2 & Biofertilizantes & 33.95 \\
3 & Abono natural & 50 \\
4 & Uso de semillas criollas & 14.81 \\
5 & Uso de semillas mejoradas o certificadas & 69.14 \\
6 & Uso de semillas transgénicas & 20.99 \\
7 & Herbicidas químicos & 64.81 \\
8 & Herbicidas orgánicos & 33.95 \\
9 & Insecticidas químicos & 46.91 \\
10 & Insecticidas orgánicos & 35.8 \\
11 & Animales de tito o yunta & 5.56 \\
12 & Tractor & 80.25 \\
13 & Maquinaria agrícola para tractor & 70.37 \\
14 & Estudios de salinidad de suelo & 35.8 \\
15 & Estudios de detención de plagas & 44.44 \\
16 & Certificación orgánica & 0 \\
\hline
\end{tabular}

En ese sentido, la práctica común sigue inclinando la balanza y el daño a los suelos, incrementándose al efecto. En el estudio se muestran datos donde solo 35.8\% de los agricultores lleva a cabo estudios de salinidad de suelos, así mismo, en cuando el uso de herbicidas e insecticidas orgánicos representados cada uno de estos con un $33.95 \%$ y $35.8 \%$ respectivamente, que son consideradas como prácticas sustentables. Referente al uso de infraestructura para apoyar la producción, 51\% de los productores manifiesta la nula participación en infraestructura de apoyo, $41 \%$ desconocía o no sabía sobre el uso de esta en su parcela, 3\% tiene una parte de invernadero, $2 \%$ vivero, $1 \%$ ambos y $2 \%$ otro tipo de construcción.

La FAO, en su documento sobre los factores que favorecen y limitan la implementación de buenas prácticas agrícolas, sostiene que se requiere de nuevas e innovadoras formas de producción, teniendo como resultado, posibles incrementos en costos, disminución de rendimientos por el uso menos intensivo de sustancias químicas, entre otros. De igual forma, la reducida infraestructura y el apoyo de las instituciones públicas puede ser una limitante en la adopción correcta de las mismas, así como un factor determinante en los países en vías de desarrollo (FAO, 2012b).

Las experiencias que el agricultor ha adquirido a lo largo de esta milenaria actividad y que hoy se ven representadas en la práctica agrícola es, sin duda, un elemento que se debe analizar a profundidad a fin de que esta relación presente patrones y comportamientos innovadores acorde a las necesidades de nuestro entorno natural, así como las de la sociedad, algo que, cuando se logre consensar, conducirá al desarrollo sustentable, definido como un proceso en el que factores 
económicos, sociales y ambientales, deben integrarse y gestionarse adecuadamente dentro de una sociedad, con el fin de crear los espacios propicios para la vida humana (Rosen et al., 2014; Zarta-Ávila, 2018).

\section{Desarrollo rural, sustentabilidad y calidad de suelos}

Existe una enorme brecha entre los logros de las zonas urbanas de una región y lo alcanzado por las áreas rurales de las mismas, nos referimos al desarrollo alineado con la calidad de vida, a la que pueden tener acceso los distintos grupos sociales de estos lugares. Analizar el desarrollo rural, significa abordar ideas evolucionistas y unidireccionales de desarrollo del territorio rural, de igual manera, se caracteriza a las zonas rurales con un tipo de desarrollo institucionalizado, donde el estado y algunos actores sociales juegan un papel muy importante (Herrera-Tapia, 2013).

El desarrollo rural sustentable se relaciona con el manejo correcto de un término conocido como agro-ecosistema, dentro del cual interactúan los sistemas social, ambiental y natural, generándose una actividad bajo una dinámica socioeconómica con fenómenos relacionados con el uso eficiente los recursos. 'El desarrollo sustentable es un desarrollo que satisface las necesidades del presente sin disminuir la habilidad de las generaciones futuras de satisfacer las suyas' (ONU, 1987).

Contrario a lo planteado por diversos autores y organismos como (Altieri y Nicholls, 2000; Sotomayor et al., 2011; Altieri, 2012; Guzmán et al., 2016; CELAC, 2017; ONU, 2019) que señalan que la sustentabilidad en los sistemas de producción agrícola, así como la agroecología serán fundamentales para redirigir el acontecer del sector rural campesino, ayudando esto a mitigar los problemas que, en sus investigaciones, señalan la degradación de los suelos, contaminación de agua y aire, entre otros, un estudio hecho por (Martín et al., 2018) plantea que, en 20 años de explotación agrícola intensiva, las propiedades de fertilidad de los suelos se mantuvo (aumento del $\mathrm{pH}$, la CIC, el $\mathrm{K}_{2} \mathrm{O}, \mathrm{P}_{2} \mathrm{O}_{5}$, Ca y $\mathrm{Mg}$ del suelo).

Ramírez- Mocarro (1998) destaca la importancia de adoptar nuevos enfoques y patrones de desarrollo rural como guía para disminuir las desigualdades y condiciones socioeconómicas, mejorar la integración territorial entre las comunidades para enfrentar y resolver la problemática ambiental y apoyar el crecimiento económico sostenido y sustentable. De acuerdo con la Oficina de Estudios y Políticas Agrarias (ODEPA, 2016) la sustentabilidad y el desarrollo alineado a este factor, se han venido agregando a la agenda política internacional de las naciones, logrando posicionarse en el discurso político, dentro de lo cual ha figurado la agricultura y sus patrones de producción; sin embargo, se ha observado que esta actividad ha causado una importante degradación de los suelos.

La Ley de Desarrollo Rural Sustentable del Estado de Sinaloa (2013) tiene por objeto promover el desarrollo rural sustentable del Estado, en uno de sus apartados hace referencia al diseño y promoción de las prácticas productivas con orientación a la conservación y mejoramiento de las condiciones de los recursos naturales, como el suelo y el agua, la biodiversidad, la inocuidad alimentaria y los servicios ambientales del campo. Se puede constatar que el desarrollo sustentable se promueve desde las leyes, mas no en las actividades reguladas, la suficiencia en este rubro no hace la diferencia, los hechos en los diversos contextos hablan por sí solos y son, para muchos, devastadores, donde la actividad del sector agrícola no es la excepción. 
Los espacios rurales son de los más vulnerables a la explotación primaria, no solo por las características propias del entorno en cuanto a su principal actividad, sino, por los elementos que caracterizan a su población y a la falta de soporte por parte de las autoridades (gobernanza ambiental), que son quienes deben fomentar la participación conjunta con la sociedad rural y la búsqueda del desarrollo idóneo para estas áreas, en ocasiones, son olvidadas por la sociedad. De tal forma que, el primer papel de la agricultura es mantener los ecosistemas; cambiando así la agricultura de una fuente de degradación a un impulsor de la restauración y la salud de los ecosistemas (ONU, 2017). Este mismo organismo apunta que recursos como el suelo, deberán ser manejados de manera más coordinada y eficiente.

Para el caso de la zona de estudio, los retos no son mínimos, los espacios rurales definitivamente presenta retrasos que, en estos lugares se pueden palpar que son muchos, si nos enfocamos a lo relacionado con el desarrollo sustentable y su relación con los factores productivos en la actividad agrícola (explotación de los recursos como los suelos y el agua), podremos darnos cuenta de que, lo que se ha estado haciendo por décadas, no ha sido lo correcto por ninguno de los actores involucrados, públicos o privados, por lo menos, no en su mayoría, sobre todo en aquellos que dedican su actividad al beneficio económico y no al consumo familiar.

De tal forma que, el desarrollo sustentable del sector agrícola sinaloense representa uno de los principales retos para los actores que en ella intervienen, debido a que su actividad es intensiva y sus patrones de producción son una mezcla de manejos tradicionales y modernos, que, si bien han sido determinantes para la actividad económica, también se ha caracterizado por los grandes problemas ambientales que dicha actividad ha originado. Pero ¿cómo se puede definir o conceptualizar el desarrollo sustentable? ¿qué tan importante es hoy en día este término para el bienestar de las regiones y de la sociedad? Sobre todo, para los asentamientos o grupos de personas que habitan en los espacios rurales y que tienen como principal actividad económica-productiva la agricultura.

Además, ¿Por qué muchas de las regiones del mundo están en la búsqueda constante, por lo menos en el discurso político, de alcanzar dicho desarrollo? ¿qué efectos genera sobre los recursos naturales como el suelo, la sobreexplotación e inapropiado uso de este? quizás las respuestas a esta serie de cuestionamientos pueden encontrarse en estudios e investigaciones de distintos académicos, en fuentes de información de estudios de instituciones de gobierno, en informes de organismos internacionales, etc.; sin embargo, las respuestas idóneas están en las comunidades rurales, en su acontecer local diario, en las necesidades que no son atendidas por el productor agrícola, quien, con sus conocimientos tradicionales, pone en práctica una actividad tan noble como la agrícola, falto de infraestructura tecnológica y financiamiento para una mejor actividad, pero haciendo parte de su trabajo como agente económico y social, que a su vez, muchas veces desconoce profundamente los efectos que puedan generarse de la inapropiada práctica de la actividad, debido a la falta de conocimientos o, simplemente lo deja de lado, pues no tiene más opción para sobresalir y cubrir sus necesidades y las de su familia.

De esa manera, los agricultores de la región estudiada han usado, por décadas, los métodos tecnológicos que, a su propio entender, se ven más como fortalezas, pero que es importante señalar que dichos métodos y formas de practicar la actividad agrícola, también se traduce en desequilibrios ecológicos regionales, que se respaldan en la revolución verde y en la bioingeniería (Aguilar-Soto y Aguilar-Aguilar, 2008). Un claro ejemplo del impacto de las prácticas agrícolas 
inapropiadas y de las externalidades negativas de la actividad a su propio entorno, es el daño irreversible causado a los suelos. El territorio nacional cuenta con 21 de los 25 tipos de suelos que existen en el mundo; 10 de ellos cubren $90 \%$ de la superficie nacional, y dentro de los más importantes destacan los siguientes (Cuadro 2).

Cuadro 2. Tipos y principales características del suelo en México.

\begin{tabular}{cccc}
\hline & \multicolumn{3}{c}{ Tipo de suelo } \\
\cline { 2 - 4 } & Regosol & Litosol & Xerosol \\
\hline Características & $\begin{array}{c}22 \% \text { del territorio } \\
\text { nacional }\end{array}$ & $17 \%$ del territorio nacional & $\begin{array}{c}12 \% \text { del territorio } \\
\text { nacional }\end{array}$ \\
Apto para & $\begin{array}{c}\text { Plantaciones de } \\
\text { árboles frutales y } \\
\text { pastoreo }\end{array}$ & $\begin{array}{c}\text { Agricultura de sustentación, } \\
\text { pastoreo y plantaciones } \\
\text { forestales }\end{array}$ & $\begin{array}{c}\text { Agricultura intensiva } \\
\text { si se cuenta con agua }\end{array}$ \\
\hline
\end{tabular}

Elaboración con datos del boletín semanal número 4 sobre el suelo del Servicio de Información Agroalimentaria y Pesquera (SIAP) de la Secretaría de agricultura, ganadería, desarrollo Rural, Pesca y Alimentación (SAGARPA, 2013).

Con datos extraídos de la entrevista aplicada, los tipos de suelos predominantes en las parcelas de los agricultores de Guasave, Sinaloa, son los siguientes (Cuadro 3).

Cuadro 3. Tipos de suelo en las parcelas en la zona de estudio.

\begin{tabular}{ccc}
\hline Número & Tipo de suelo & $(\%)$ \\
\hline 1 & Arenoso & 30.86 \\
2 & Arcilla & 23.46 \\
3 & Aluvión & 14.2 \\
4 & Francos & 8.64 \\
5 & Barrial & 8.02 \\
6 & limoso & 5.56 \\
\hline
\end{tabular}

El resto de los suelos fueron representados con porcientos menores.

En la Figura 1 se describen los problemas que los agricultores mencionaron tener al momento de la práctica agrícola, para el análisis del resultado se tomaron en cuenta solamente las respuestas positivas para cada componente estudiado, en donde se tiene que $25.93 \%$ tiene problemas de pérdida de fertilidad en el suelo, 59.26\% considera que los costos en los insumos es un problema predominante (compra de fertilizantes, agroquímicos, entre otros), $10.49 \%$ contestó que su parcela presenta salinidad, $74.7 \%$ no conoce el tipo de suelo de su terreno y $43.21 \%$ ha hecho algún tipo de estudio al suelo, con el propósito de conocer la calidad de estos para los cultivos.

Como se puede apreciar, la pérdida de fertilidad en los suelos es un hecho y está acompañada por la salinidad en los mismos, si a ello se le agregan variables como el no conocer el suelo predominante en la parcela y que la mayoría de los agricultores no llevan a cabo estudios de suelo para conocer la problemática a la que se enfrentan directamente, aun cuando $43.21 \%$, de los entrevistados considera que es muy importante realizar estudios a la tierra para conocer su grado de calidad, esto hace que el problema sea aún mayor y el actuar del campesino, sigue siendo con base en su experiencia y no, el aplicar estrategias tecnológicas y ciencia a su actividad dentro del sector y de su predio agrícola. 


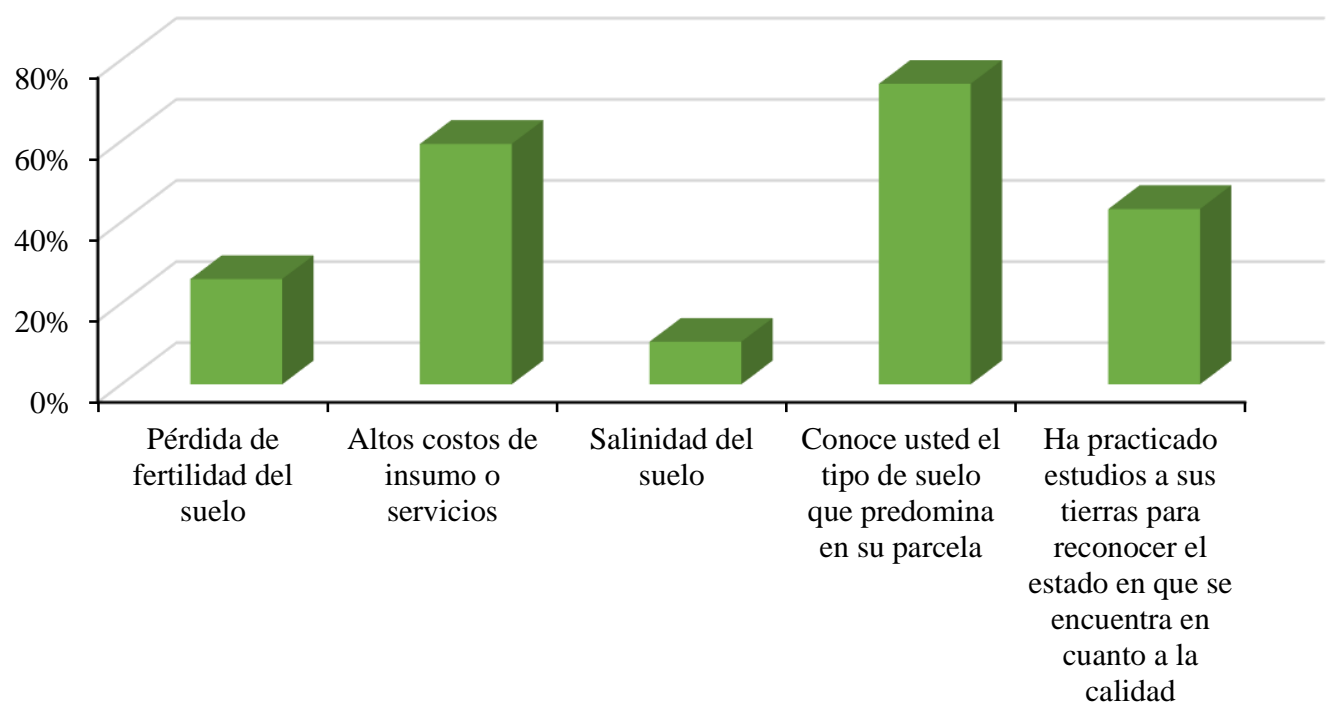

Figura 1. Principales problemas que tuvo el agricultor para el desarrollo de la actividad agrícola.

Para la Secretaría de Medio Ambiente y Recursos Naturales (SEMARNAT) y el Colegio de Postgraduados (CP), en México, el proceso más importante que origina la degradación de los suelos es la erosión hídrica, los estados con mayor afectación por la salinización es causada por la degradación química que está representada dentro de las prácticas agrícolas tradicionales, y en estos rubros está Sinaloa con un (3.5\%), Guanajuato con (3.3\%) y Tamaulipas con (2\%) de la superficie (SEMARNAT-CP, 2003).

En el Cuadro 4 se muestran datos del Instituto Nacional de Estadística y Geografía (INEGI, 2007) del censo agropecuario. Se puede apreciar que la práctica agrícola se apoya principalmente en la fertilización, uso de herbicidas e insecticidas químicos, quedando los abonos naturales con menor importancia de aplicación.

Cuadro. 4. Unidades de producción con superficie agrícola y tipo de tecnología o práctica que se usa en el estado de Sinaloa.

\begin{tabular}{ccc}
\hline Número & Unidades de producción & 40187 \\
\hline 1 & Fertilizantes químicos & 32201 \\
2 & Semilla mejorada & 27054 \\
3 & Abonos naturales & 2428 \\
4 & Herbicidas (total) & 19962 \\
5 & Herbicidas químicos & 19249 \\
6 & Herbicidas orgánicos & 1739 \\
7 & Insecticidas (total) & 20750 \\
8 & Insecticidas químicos & 20596 \\
9 & Insecticidas orgánicos & 552 \\
10 & Quema controlada & 2298 \\
11 & Otras tecnologías & 67 \\
\hline
\end{tabular}

Elaboración con datos del (INEGI, 2007). 
En ese sentido, se les preguntó a los agricultores bajo qué criterios utilizaba o ponía en práctica actividades como las descritas en la cuadro anterior, $56.79 \%$ de los entrevistados comenta que solicita la ayuda de un agrónomo, $51.85 \%$ lo hace con base en el conocimiento empírico (experiencia), mientras que $16.67 \%$ busca la ayuda de otro agricultor y $12.35 \%$ recibe ayuda de los vendedores; si se suma lo empírico y la ayuda entre agricultores, esto da $68.52 \%$, lo cual indica que la experiencia sigue siendo la base del comportamiento del agricultor en su práctica agrícola.

Como se puede observar, si los problemas derivados de las prácticas agrícolas inapropiadas que afectan la sustentabilidad de los suelos fuesen pocos, a esto se agrega lo causado por el cambio climático, efectos que para muchos pueden ser devastadores, empezando por la baja producción y repercusión en la seguridad alimentaria de millones de personas que habitamos este planeta, pero es tema de otro trabajo de investigación, solo se menciona como parte de un efecto dominó.

\section{Conclusiones}

La situación actual del entorno agrícola es un efecto del contexto mundial, la globalización de los mercados y de las economías, esto ha reestructurado el quehacer de los agentes económicos y de las actividades económicas preponderantes de cada una de las regiones de un país y del mundo, resultando esto en una serie de factores que lastiman el bienestar de la sociedad y que en su conjunto, estas han depredado el entorno natural en el que se desarrollan y coexisten, llevando así, a la falta de desarrollo dentro del marco de la sustentabilidad.

La actividad agrícola representa para el municipio de Guasave y la región noroeste del estado de Sinaloa una de las principales bases económico-productivas, ya que es de las más fuertes, económicamente hablando, en ese sentido, la falta de sustentabilidad del sector rural, ha sido uno de los efectos más severos que se originan del actuar de dichos agentes económicos, al igual que la calidad de los suelos cada vez más pobres, principalmente debido a la inadecuada práctica agrícola, misma que tiene como origen el comportamiento tradicional del campesino o la falta de conocimiento técnico-productivo sobre su principal actividad dentro de su parcela.

La presión que genera la actividad productiva sobre el medio ambiente se encuentra relacionada, con la necesidad de producir los bienes con mayor rendimiento, ya que, los bajos precios de los productos, generan la necesidad de buscar mayor intensidad productiva, tanto para conseguir los bienes indispensables, como para incrementar los beneficios de la comercialización. Este último objetivo ha alcanzado fuerte influencia en las décadas recientes, con la libertad de las empresas y menor control del Estado, característica del modelo capitalista vigente en la mayor parte del mundo. En tales condiciones, se requiere equilibrar esos extremos, donde se lleve mayor control de las instituciones hacia las empresas y además, el mejoramiento de los ingresos y servicios de la población, para que se encuentre en condiciones de exigir los requisitos de calidad de los alimentos, reglamentando a las empresas en las tecnologías amigables del ambiente, para relajar la presión de sobreexplotación de los recursos y emisión de contaminantes.

Si se quieren ver cambios positivos de los recursos naturales como el caso del suelo, urge un cambio en la estructura técnico-productiva del sector agrícola, además de una modificación a los patrones de comportamiento de los agentes productivos del sector y se requiere mejorar los aspectos legales que regulan el uso de insumos agrícolas que deterioran los recursos naturales disponibles y el bienestar de la sociedad (gobernanza ambiental) o los daños a corto o mediano plazo serán irreversibles y catastróficos en términos sustentables: ambientales, económicos y sociales. 


\section{Literatura citada}

Aguilar-Soto, O. A. y Aguilar-Aguilar, G. 2008. Competitividad agrícola y granos en el Noroeste de México: el caso de Sinaloa. 1-18 pp.

Altieri, M. A. 2012. Agroecología: principios y estrategias para diseñar una agricultura que conserva recursos naturales y asegura la soberanía alimentaria. Universidad de California, Berkeley. 1-192 pp.

Altieri, M. y Nicholls, C. I. 2000. Teoría y práctica para una agricultura sustentable $1^{\text {st }}($ Ed.). ONUPNUMA. 215-231 pp.

Andrade, V. H. G. Z.; Redmile-Gordon, M., Barbosa, B. H. G.; Andreote, F. D.; Roesch, L. F. W. and Pylro, V. S. 2021. Artificially intelligent soil quality and health indices for 'next generation' food production systems. Trends Food Sci. Technol. 107:195-200. https://doi.org/10.1016/j.tifs.2020.10.018.

Arias, Fi. 2012. El proyecto de investigación introducción a la metodología científica. $6^{\text {ta. }}$ Edición. EPISTEME. Caracas.68-73 pp.

Baveye, P. C.; Baveye, J. and Gowdy, J. 2016. Soil "ecosystem" services and natural capital: critical appraisal of research on uncertain ground. Front. Enviro. Sci. 4(41):1-40. https://doi.org/10.3389/fenvs.2016.00041.

CEDRSSA (Centro de Estudios para el Desarrollo Rural Sustentable y la Soberanía Alimentaria). 2019. El suelo, un recurso invaluable para la producción de alimentos. Estudios e investigaciones. Cámara de Diputados. 1-18 pp.

Castillo, B.; Ruiz, J. O.; Manrique, M. A. y Pozo, C. 2020. Contaminación por plaguicidas agrícolas en los campos de cultivos en Cañete (Perú). Espacios. 41(10):1-20.

Castillo-Valdez, X.; Etchevers-Barra, J. D.; Hidalgo-Moreno, C. M. I. y Aguirre-Gómez, A. (2021). Evaluación de la calidad de suelo: generación e interpretación de indicadores. Rev. Terra Latinoam. 39:1-12. https://doi.org/10.28940/terra.v39i0.698.

CELAC (Comunidad de Estados Latinoamericanos y Caribeños). 2017. Sistemas de innovación para el desarrollo rural sostenible. Organización de las Naciones Unidas para la Alimentación y la Agricultura (FAO). 15-29 pp. http://www.fao.org/3/a-i7769s.pdf.

Chalán, J. M. 2019. Agricultura convencional y agroecología frente al cambio climático. Universidad Andina Simón Bolívar. 17-18 pp.

CODESIN (Consejo para el Desarrollo Económico de Sinaloa). 2019. Agricultura en Sinaloa 2018. https://sinaloaennumeros.codesin.mx/wp-content/uploads/2019/07/Brief-Agricultura-enSinaloa-2018.pdf.

CONAFOR (Comisión Nacional Forestal) y UACH (Universidad Autónoma de Chapingo). 2013. Línea base nacional de degradación de tierras y desertificación. http://www.semarnat.gob.mx/sites/default/files/documentos/fomento/documentos/degrada cion-tierras-desertificacion2.pdf.

Díaz, A.; Gebler, L.; Maia, L.; Medina, L. y Trelles, S. 2017. Buenas prácticas agrícolas para una agricultura más resiliente. Lineamientos para orientar la tarea de productores y gobiernos. Instituto Interamericano de Cooperación para la Agricultura. https://www.redinnovagro.in/ pdfs/bve17069027e_Gu\%C3\%ADa.

FAO (Organización de las Naciones Unidas para la Alimentación y la Agricultura). 2012a. Factores que favorecen y limitan la implementación de las buenas prácticas agrícolas en la cadena hortícola. https://coin.fao.org/coin-static/cms/media/11/13305393011350/sistematizacion_ bpas_hortalizas_febrero_2012_atinar.pdf. 
FAO (Organización de las Naciones Unidas para la Alimentación y la Agricultura). 2012b. Manual de buenas prácticas agrícolas para el productor hortofrutícola. https://www.fao.org/ 3/as171s/as171s.pdf.

Fox, J. y Haight, L. 2010. La política agrícola mexicana: metas múltiples e intereses en conflicto. In Subsidios para la desigualdad: las políticas públicas del maíz en México a partir del libre comercio $1^{\text {st }}($ Ed). Wilson Center. 9-53 pp.

Gobierno de Sinaloa. 2019. Tercer informe de gobierno 2019 del estado de Sinaloa. Anexo estadístico y gráfico. https://sinaloa.gob.mx/uploads/files/tercer_informe/Tercer_Informe_ Anexo_Estad\%C3\%ADstico_y_Gr\%C3\%A1fico.pdf.

Gobierno municipal de Guasave. 2019. Agricultura en Guasave. http:/guasave.gob.mx/s/ agricultura/.

González, A.; Del-Amo, S. y Gurri, F. 2007. Los nuevos caminos de la agricultura. Procesos de conversión y perspectivas. Península. 2(2):1-12.

González-Jácome, A. 2004. Ambiente y cultura en la agricultura tradicional de México: casos y perspectivas. Ciencia Ergo Sum. 11(2):153-163.

Guzmán, P.; Guevara, R.; Olguín, J. y Mancilla, O. 2016. Perspectiva campesina, intoxicaciones por plaguicidas y uso de agroquímicos. Idesia. 34(3):69-80.

Ley de desarrollo rural sustentable del estado de Sinaloa. 2013. 143 (1): 1- 49.

Herrera-Tapia, F. 2013. Enfoques y políticas de desarrollo rural en México: una revisión de su construcción institucional. Gestión y Política Pública. 22(1):131-159.

Instituto Nacional de Estadística y Geografía (INEGI). 2007. Censo Agropecuario 2007. http://www.beta.inegi.org.mx/contenidos/proyectos/agro/agricola/2007/tabulados/Tabulad o_VIII_CAGyF_21.pdf.

Laurett, R.; Paço, A. and Mainardes, E. W. 2021. Measuring sustainable development, its antecedents, barriers, and consequences in agriculture: An exploratory factor analysis. Environ. Develop. 37:1-14. https://doi.org/10.1016/j.envdev.2020.100583.

López-Sánchez, A.; Luque-Badillo, A. C.; Orozco-Nunnelly, D.; Alencastro-Larios, N. S.; RuizGómez, J. A.; García-Cayuela, T. and Gradilla-Hernández, M. S. 2021. Food loss in the agricultural sector of a developing country: transitioning to a more sustainable approach. The case of Jalisco, Mexico. Environ. Challenges. 5(2021):1-16 https://doi.org/10.1016/ j.envc.2021.100327.

Martín, G. M.; Espinosa, R. R.; Fundora, L. R.; Cabrera, A. y Martín, N. 2018. Evolución de algunas propiedades químicas de un suelo después de 20 años de explotación agrícola. Cultivos Tropicales. 39(4):21-26.

ODEPA (Oficina de Estudios y Políticas Agrarias). 2016. Protocolo de Agricultura Sustentable. https://www.odepa.gob.cl/wp-content/uploads/2017/12/3-Protocolo-AgriculturaSustentable.pdf.

ONU (Organización de las Naciones Unidas). 1987. Informe de la Comisión Mundial sobre Medio Ambiente y el Desarrollo: nuestro futuro común. (informe núm. 42). Naciones Unidas. http://www.ecominga.uqam.ca/PDF/BIBLIOGRAPHIE/GUIDE_LECTURE_1/CMMADInforme-Comision-Brundtland-sobre-Medio-Ambiente-Desarrollo.pdf.

Organización de las Naciones Unidas (ONU). 2019. Cinco cosas que debes saber sobre la agenda 2030 para el desarrollo sostenible. https://www.onu.org.mx/5-cosas-que-debes-sabersobre-la-agenda-2030-para-el-desarrollo-sostenible/. 
Peinado-Guevara, V.; Peinado-Guevara, H.; Campista-León, S. y Delgado-Rodríguez, O. 2015. Análisis de la producción agrícola y gestión del agua en módulos de riego del distrito 063 de Sinaloa, México. Estudios Sociales: Rev. Investig. Cient. 23(46):115-136.

Pérez Sánchez, J. M.; Velasco Orozco, J. J. y Reyes Montes, L. 2014. Estudios sobre agricultura y conocimiento tradicional en México. Perspectivas latinoamericanas. 11(26):144-156.

Ramírez-Mocarro, M. A. 1998. Desarrollo sustentable en áreas rurales marginadas: entre la sobrevivencia y la conservación. Papeles de Población. 4(18):123-141.

Reus-González, N. N.; Reus-González, T.; Macías-Ocampo, M. J. and Castro-López, E. 2021. Banking cards on students of bachelor's degree in law in personal finance management. Am. J. Hum. Soc. Sci. Res. 5(4):579-586. www.ajhssr.com.

Rosen, M. A.; Dincer, I. and Hacatoglu, K. 2014. Sustainability. Encyclopedia of toxicology. Elsevier. https://doi.org/10.1016/B978-0-12-386454-3.01046-0.

SEMARNAT (Secretaría del Medio Ambiente y Recursos Naturales)-COLPOS (Colegio de Postgraduados). 2003. Evaluación de la degradación del suelo causada por el hombre en la república mexicana escala 1:250 000. Memoria Nacional.

SIAP (Servicio de Información Agroalimentaria y Pesquera). 2020. Avance de siembras y cosechas: resumen por estado. http://infosiap.siap.gob.mx:8080/agricola_siap_ gobmx/resumenproducto.do.

Sotomayor, O.; Rodríguez, A. y Rodríguez, M. 2011. Competitividad, sostenibilidad e inclusión social en la agricultura: Nuevas direcciones en el diseño de políticas en América Latina y el Caribe. CEPAL. Santiago, Chile. 255-267 pp.

Tamayo, M. J. M.; Cruz C. C. y Munguía, G. A. 2016. Los Conocimientos Tradicionales y la Agricultura Moderna: Caso Dzidzantún, Yucatán y Huatusco, Veracruz. El desarrollo regional frente al cambio ambiental global y la transición hacia la sustentabilidad. (Ed.). 1a. Asociación Mexicana de Ciencias para el Desarrollo Regional A. C. México.

Taylor, S. J. y Bogdan, R. 1984. Introducción a los métodos cualitativos. (Ed.). ${ }^{\text {ra }}$. Editorial Paidós. 31-46 pp.

Valenzuela-Martínez, C.; Romano-Casas, G.; Cuadras-Berrelleza, A. A. y Ortega-Martínez, L. D. 2019. Plaguicidas, impacto en salud y medio ambiente en Sinaloa (México): implicaciones y retos en gobernanza ambiental. Trayectorias humanas transcontinentales. 4:103-122. https://doi.org/10.25965/trahs.1615.

Viana, C. M.; Freire, D.; Abrantes, P.; Rocha, J. and Pereira, P. 2022. Agricultural land systems importance for supporting food security and sustainable development goals: A systematic review. Sci. Total Environ. 806(3):1-9. https://doi.org/10.1016/j.scitotenv.2021. 150718.

Zarta-Ávila, P. 2018. La sustentabilidad o sostenibilidad: un concepto poderoso para la humanidad. Tabula Rasa. 28:409-423. https://doi.org/10.25058/20112742.n28.18.

Zinck, J. A.; Berroterán, J. L.; Farshad, A.; Moameni, A.; Wokabi, S. y Van Ranst, E. 2005. La sustentabilidad agrícola: un análisis jerárquico. Gaceta Ecol. 76:53-72. 